\title{
Epigenetic control of apomixis: a new perspective of an old enigma
}

\begin{abstract}
Apomixis is an asexual mode of reproduction through seeds in plant which results in clonal multiplication of the maternal plant due to the absence of meiosis and fertilization of egg cell. It has been reported to occur in $>300$ plant species belonging to $>35$ angiosperm families. Apomixis has been common in polyploid grasses, and poorly represented in the crop species. If apomixis could be incorporated in crop plants, its impact on agriculture could be overwhelming because apomixis promises to maintain hybrid vigor in the progenies over the generations. However, engineering apomixis in crop plant is considered to be one of the greatest challenges mainly because of the limited knowledge about the genetic basis and regulatory mechanisms underlying the apomictic seed development. The genetic and molecular bases of apomixis is still intriguing from both developmental and evolutionary perspectives. Coexistence of apomixis and sexuality in an individual plant suggests that apomixis is reversibly superimposed upon sexuality. Epigenetic control of apomixis is an attractive and emerging theory being investigated with growing support from the studies on sexual plants wherein mutations in epigenetic pathway genes resulted into apomixislike phenotypes. With the growing evidences for epigenetic variations as important regulator of stress-associated genes, and the evidence for upregulation of stressassociated metabolite (spermidine and polyamine) biosynthesis, epigenetics is likely to be in focus of future studies on apomixis. Therefore, apomixis could be introgressed into crop plants in future by switching-off the sexual pathway to an apomixis-like route by transferring the candidate gene(s). However, flexibility to switch apomixis/ sexuality on and off would be necessary for crop breeding purposes.
\end{abstract}

Keywords: apomixes, epigenetics, DNA methylation, gene regulation, crop improvement, histone modification, asexual reproduction, seed development
Volume 7 Issue I - 2017

\author{
Suresh Kumar \\ Division of Biochemistry, ICAR-Indian Agricultural Research \\ Institute, New Delhi-I I00 I2, India
}

\begin{abstract}
Correspondence: Suresh Kumar, Division of Biochemistry, ICAR-Indian Agricultural Research Institute, New Delhi-I I00 I2, India Phone +91 I-I25-842-038, Fax +9| I-I25-846-420,
\end{abstract} Email sureshkumar3_in@yahoo.co.uk

Received: February 16, 2017 | Published: May 02, 2017

\section{Introduction}

Apomixis (asexual mode of reproduction through seeds) results into the production of progenies genetically identical to the female parent. It is a unique, naturally-occurring mode of reproduction in plant which produces seed without meiosis and/or fertilization of the egg cell. To understand the difference between sexual and apomictic seed development pathways, it is necessary to first look at the events required for sexual seed development. Seed formation in flowering plants (angiosperms) begins with the switching from vegetative to reproductive growth in the plant. This leads to the formation of flower containing male (anther) and female (ovule) reproductive organs which produce diploid gamete-precursor cells. Male gameteprecursor cells differentiate into anther, while a single female gameteprecursor cell differentiates into megaspore mother cell in ovule. Male and female gamete-precursor cells undergo meiosis followed by mitosis to form mature multicellular male and female gamete. Seed development initiates with the fertilization of ovule with pollen, containing two sperm cells. One of the sperm cells fuses with the egg cell in the ovule (that gives rise to the diploid embryo), while the other sperm cell fuses with the diploid central cell to form triploid endosperm (which provides essential nutrients to the developing embryo). Tissues surrounding the developing embryo and endosperm form seed coat of the mature seed. In contrast, apomixis can occur by various mechanisms that share three common developmental components:

i. bypassing meiosis during embryo sac formation (apomeiosis) ii. development of embryo without fertilization (parthenogenesis)

iii. Formation of endosperm either without fertilization or fertilization with sperm cell.

Thus, the major events that characterize apomixis include: avoidance of meiotic reduction during embryosac formation, fertilization-independent embryo development, and the formation of endosperm with/without fertilization. ${ }^{1}$ Apomixis may occur by different pathways, the most common is the gametophytic apomixis which occurs through the formation of an unreduced female gametophyte. In diplospory, the progenitor cell of the unreduced embryosac is the megaspore mother cell, whereas in apospory, a somatic nucellar cell or even integument differentiates to form an aposporous initial (AI) and initiates gametophytic development. In both the cases, the egg cell develops parthenogenetically. While in many of the grasses, either fertilization of the central cell is required for apomictic seed development (pseudogamy) or the central cell develops autonomously to produce the endosperm.

Apomixis not only provides a means of clonal propagation through seeds (because the progenies produced through apomixis are genetically identical to the mother plant), but also makes genetic improvement of the species difficult and time consuming. It restricts varietal development process to selection of elite lines from the natural variants only in the apomictic species, and no other breeding approach works. ${ }^{2-3}$ Induction of parthenogenesis by transferring specific gene(s) to a crop plant may be a step towards synthesizing 
apomixes. ${ }^{4}$ Apomixis can be combined with haploid induction to rapidly obtain homozygous lines in breeding programs. Genes for the components of apomixis have been reported to be tightly linked in majority of apomictic species. ${ }^{5}$ Evidences indicate that the components of apomixis can be uncoupled by recombination and epigenetics. ${ }^{6}$ However, even after considerable efforts during the last four decades, success in transferring apomixis to a crop species has not yet been materialized, mainly because all the gene(s) for the components of apomixis have not yet been identified, cloned and characterized.,

\section{Spread of apomixis in plants}

Apomixis has been reported in more than 35 angiosperm families and in over 300 plant species, $75 \%$ of which belong to three families viz. Poaceae et al. ${ }^{8}$ Unfortunately, barring a few exceptions from some forage grasses and fruit trees, apomixis is not common in crop species. It is believed that it occurs mostly in polyploid genotypes. ${ }^{9}$ Interestingly, Kumar et al., ${ }^{10}$ identified a rare obligate sexual, polyploid (tetraploid) plant of Cenchrus ciliaris. However, discovery of apomixis in diploid species ${ }^{11}$ proves that polyploidy is not an absolute necessity for apomixes. ${ }^{12}$ Recently, Kumar et al., ${ }^{8}$ reported no correlation between the mode of reproduction (apomixis) and ploidy level (polyploid) or habit (perennial) in the Cenchrus spp. available in India. While four apomictic Cenchrus spp. were reported to be perennial in nature, C. myosuroides (a perennial and polyploidy species) was found to be obligate sexual. They also reported two polyploid species (C. echinatus and C. myosuroides) to be sexual, and a diploid species (C. setigerus) to be apomictic. All these indicate that polyploidy is not an absolute requirement for apomixis. Thus, occurrence of apomixis in polyploids is still debated with a strong yet poorly understood correlation between apomixis and polyploidy. ${ }^{8,13,14}$

In spite of the expected faithful transmission of maternal genotype to the progenies, sufficient genetic variability has often been observed in apomictic populations. ${ }^{15}$ At least three different processes have been proposed to contribute to the genetic variability. ${ }^{16}$ First is the build-up of genetic variations in apomictic individual due to the accumulation of new mutations, just like it occurs in any sexual plant. Second may be the leaky-process of apomixis, wherein the apomictic mode of reproduction is supplemented by a small and often irregular amount of sexuality which may add new/recombinant genotypes to the population. A third process for creating genetic variation in an apomictic population may be the facultative mode of reproduction. All of these processes have been widely discussed and analyzed by different researchers. ${ }^{3,17-19}$

\section{Breeding for apomixis}

Although considerable yield enhancement has been recorded due to the adoption of Green Revolution technologies including high-yielding, input-responsive hybrid varieties of crops, efforts are continuously being made to further increase productivity of crop plants to feed the burgeoning global population. The global population is expected to reach 9billion by 2050 with the growth of 2-3billion people over the next 35years. ${ }^{20}$ To feed the ever-growing global population, we need to produce more food and livelihood opportunities from less per capita arable land and water (i.e. more from the less). There are several challenges in sustainably feeding the growing global populations. Over the centuries, plant breeding has followed the general pattern of introduction, selection and hybridization. Once an elite genotype is introduced, selection and breeding strategies lead towards the development of new varieties for enhanced yield/quality.
Clonal multiplication of a superior genotype has been successful via vegetative propagation; however, it does not work in case of annual crops like rice, wheat and maize. Interestingly, fixing of genotype occurs naturally in certain plant species through apomictic mode of production. Theoretically, apomixis can be introgressed into desired crop plant through conventional breeding, but the process is very slow (time-consuming) and laborious at field level. This requires embryological/progeny analysis of a huge breeding population for several generations to select apomictic genotypes after each round of backcrossing. ${ }^{21}$ Moreover, there are several breeding constraints including the availability of desirable/appropriate parental lines and efficient techniques (including molecular markers) for screening the segregating populations. Obligate sexual plant, which can be used as female parent, is one of the prerequisites for this. However, occurrence of obligate sexual genotype is rare because over a long period apomictic individuals outnumber the sexual one. Nevertheless, obligate sexual plant in different apomictic species has been identified in the natural population. ${ }^{10,22,23}$ A natural variant of Indian accession of buffelgrass was identified and reported to be obligate sexual, protogynous and self-incompatible in nature, ${ }^{24}$ which could be an appropriate material for cross-hybridization in breeding for apomixis.

Successful use of apomixis in crop breeding requires deeper knowledge of the mechanisms regulating reproductive development in plants. Our molecular understanding of apomixis can be greatly improved if molecular markers and the genes/pathways involved in the development of embryo and embryosac could be identified. A large amount of cytological, ecological, embryological, genetic and molecular data are becoming available from several apomictic species, ${ }^{8,10,25,26}$ some of the candidate genes have been tested in Arabidopsis thaliana to elucidate the mechanisms of apomeiosis, parthenogenesis and apomixis. However, none of them could mimic the apomixis process. Newer concepts are being proposed to link apomixis with regulation of reproductive pathways, including chromatin remodeling (epigenetics), apomixis-bearing chromosome (accumulated with transposable elements), role of auxin in deciding fate of the embryosac and the egg cell development, ${ }^{27}$ which require proper validation at least in the model plant system.

\section{Genetics and molecular biology of apomixis}

Evidences for single dominant locus responsible for apomeiosis and parthenogenesis are available from aposporous grasses including Pennisetum/Cenchrus, ${ }^{28}$ Brachiaria, $^{29}$ Paspalum $^{30}$ and Tripsacum. ${ }^{31}$ With the advent of DNA-based molecular markers, a new generation of markers has been introduced which has revolutionized research in the biological sciences. Since the development of DNAbased markers, they are continuously being modified/improved to enhance their efficiency, and being utilized to bring about automation in the process of genome analysis. ${ }^{32}$ Different types of DNA markers are available now to analyze polymorphism as well as to tag the gene of interest. Among these, simple sequence repeat (SSR) marker has been developed more recently, and the marker is expected to bring even more rapid advances in markers development and their use in crop breeding programs. ${ }^{33}$ To better understand the process of apomixis, molecular markers linked with apomictic/sexual mode of reproduction are necessary in handling huge segregating populations. ${ }^{3}$ Studies based on molecular markers indicate that there is an aposporyspecific genomic region (ASGR) in Pennisetum/Cenchrus is which is physically large, hemizygous and heterochromatic. ${ }^{34}$ The available data on apomictic mode of reproduction in plants indicate that 
apomixis is under simple genetic control in C. ciliaris. ${ }^{3,19,34,35}$ Poa pratensis, ${ }^{36}$ Panicum maximum, ${ }^{37}$ Pennisetum squamulatum, ${ }^{28,38,39}$ Brachiaria decumbens, ${ }^{29}$ Paspalum notatum ${ }^{30}$ and Tripsacum dactyloides. ${ }^{40}$ Apomixis bypasses meiotic reduction of chromosomes and fertilization of egg cell with pollen to exclude sexual processes towards production of the progenies that are genetic clone of the female parent. This happens in case of obligate apomicts, but those that do not totally exclude sexual reproduction are called facultative because they produce seeds through both sexual as well as apomictic means. Growing evidences suggest that apomixis and sexual reproduction share some key regulatory mechanisms; ${ }^{41}$ therefore, some of the researchers believe that the line between apomixis and sexuality is ambiguous. This also indicates that apomixis might have resulted from deregulation of sexuality, rather than its emergence as a novel mode of reproduction.

The molecular markers reported to be linked with sexual and apomictic modes of reproduction ${ }^{8,10,42,43}$ may be utilized for screening of segregating population, mapping of genes responsible for the mode of reproduction and their characterization using a reverse genetics approach. ${ }^{44}$ Identification of molecular markers for apomixis ${ }^{8,19,42}$ and sexuality, ${ }^{43}$ development of mapping/segregating populations, ${ }^{3,19}$ standardization of plant regeneration ${ }^{45-47}$ and genetic transformation protocols ${ }^{48,49}$ are some of the efforts made by the author to understand the process of apomixis in Cenchrus ciliaris. Further, gametophytic apomixis has been reported to be genetically controlled by two or more linked loci in different aposporous and diplosporous species. ${ }^{28}$ In $C$. ciliaris, there are evidences for simple genetic control of apomixes. ${ }^{3,19}$ Apomictic C. ciliaris reproduces by apospory which is characterized by apomeiosis and parthenogenesis. Recently, ASGR-BABY BOOMlike (PSASGR-BBML) gene from Pennisetum squamulatum (L.) R.Br. was reported to express in egg cells before fertilization and induced parthenogenesis, thus produced haploid offsprings in transgenic sexual pearl millet. ${ }^{4}$ Spatial and temporal shift in the expression of the genes of reproductive pathways in sexual and apomictic plants has been reported. ${ }^{50}$ Epigenetic regulatory mechanisms can be used to explain such deregulation. Although recent studies reveal transcriptional basis of the female germline development in sexual model plant Arabidopsis thaliana,${ }^{51}$ only little is known about the epigenetic regulation of apomixis.

\section{Epigenetics and gene regulation}

There are growing evidences that chromatin modifications, small inhibitory RNA (siRNA) and DNA methylation are involved in regulation of gene expression at transcriptional and posttranscriptional levels. Epigenetics refers to the heritable variations in phenotypic/gene expression pattern resulting from the modification of DNA, histones and small-RNA biogenesis without changing the underlying nucleotide sequences. ${ }^{52}$ Among the above epigenetic regulatory mechanisms, DNA methylation is considered to be the best understood..$^{53}$ DNA methylation and histone modifications are influenced by various abiotic and biotic factors, resulting into better adaptability of the plants to the adverse environmental conditions. DNA methylation has been reported to play a key role in gene expression through RNA-directed DNA methylation (RdDM) of genes as well as by inducing histone modifications. Methylated cytosine $(5-\mathrm{mC})$ has been reported to be involved in many important biological processes, including movement of transposable elements (TEs), genome imprinting and regulation of gene expression. ${ }^{54}$ Methylated cytosine, also known as the fifth base, was identified long before the DNA was recognized as the genetic material. Content of 5-mC in genomic DNA varies considerably among eukaryotes. The nuclear genome of higher plants may contain more than $50 \%$ $5-\mathrm{mC}$ in all the three nucleotide sequence contexts: $\mathrm{CG}, \mathrm{CHG}$, and $\mathrm{CHH}$ (where $\mathrm{H}=\mathrm{C}, \mathrm{T}$, or $\mathrm{A}$ ). While DNA methylation is restricted to symmetric CG context in mammals, non-CG methylation is prevalent in embryonic stem cells of plant. Also, cytosine methylation in $\mathrm{CHG}$ and $\mathrm{CHH}$ context is predominant in TEs, but $\mathrm{CG}$ methylation is abundant in both TEs and the functional genes. ${ }^{55}$ In mammals and plants, centromeric and pericentromeric regions of chromosomes and the repetitive elements are heavily methylated. The level of $5-\mathrm{mC}$ is determined by DNA methylation and demethylation processes. Methylation can be removed from the DNA by active and/or passive mechanisms. Promiscuous methylation requires to be pruned by demethylases to create a desired methylation pattern. Demethylation may also be required to activate specific genes or to reset epigenetic state of the genome in response to the environmental perturbations or during the developmental stages. ${ }^{56}$ Covalent modification of histone proteins is another epigenetic mechanism to control gene expression. Histone modifications, particularly methylation of 9th lysine residue of histone H3 (H3-K9), cause chromatin condensation and block transcriptional initiations. Cytosine methylation can further reinforce histone modification patterns conducive to gene silencing. Some histone modifications such as acetylation, phosphorylation and ubiquitination have been reported to enhance gene transcription, ${ }^{57}$ while biotinylation and sumoylation have been reported to repress the gene expression. ${ }^{58}$ Dynamic histone modification may get converted into DNA methylation, which are often more stable. ${ }^{59}$

Genetic analysis of Arabidopsis mutants impaired for the genes of siRNA biogenesis pathway revealed the involvement of siRNAs in RNA-directed DNA methylation (RdDM). In Arabidopsis, a 24nt siRNA was reported to down regulate the expression of $P 5 C D H$ through mRNA cleavage, leading to reduced proline degradation, and enhanced proline accumulation resulting into salt stress tolerance. ${ }^{60}$ More studies would be required to unravel the roles of RdDM pathway in epigenetic gene regulation and their applications in epigenetic manipulation for genetic engineering. Further understanding the epigenetic mechanisms of gene regulation may not only provide basic information for regulation of stress-associated genes, but it may provide a valuable platform for efficient use of plant genetic engineering towards enhanced tolerance to environmental stresses. Therefore, deciphering epigenetic machineries of gene expression has become an important area of scientific investigations to better understand the biological processes in plants.

\section{Epigenetic control of apomixis}

Apomixis has been proposed to be evolved to let the plant species propagate under adverse environmental conditions. Reactive oxygen species (ROS) are produced in excess in plants under various environmental stresses. Although ROS are also generated in plants under optimal growth conditions, but at very low concentration. Most environmental adversaries trigger enhanced production of ROS, and plants have innate systems for scavenging/detoxifying ROS. Polyamines (putrescine, spermidine and spermine) are low molecular weight, polycationic aliphatic molecules that are well-known for antisenescence and anti-stress effects due to their antioxidant properties. Spermidine is known to be involved in protection of DNA from oxidative stress by quenching free radicals arising from ROS. ${ }^{61}$ Gene for spermine/spermidine synthase has been identified to be present 
in the ASGR of $P$. squamulatum. ${ }^{62}$ Higher activity of spermidine metabolism in the AI cell was correlated with the requirements for the repair of DNA damage caused due to oxidative stress. Hence, environmental stresses and the increased levels of ROS have been considered to be major driving force for the evolution of apomixes. ${ }^{63}$ Existence of variability in developmental processes and the type of cells from which the apomixis process begins in a vegetatively propagated apomictic plant indicate that the process is not tightly regulated, and supports the possible involvement of epigenetic regulatory mechanisms in controlling apomixis process. Hypothesizing apomixis to be epigenetically regulated, comparative analyses of small-RNA, histone modifications and cytosine methylation in the apomictic and sexual individuals have been initiated. TEs make a significant proportion of genomes of higher plants and their activation/movement can have a range of effects, including genome evolution, structural and functional alterations in gene expression, gene deletion and insertion. Therefore, control on movement of TEs becomes crucial for the integrity of the host genome. TEs are generally enriched in the centromeric and telemetric regions, which are highly methylated and packed as heterochromatic regions. In maize, most of the TEs have been found to be restricted to the methylated, heterochromatin regions. This indicates that TEs and other repetitive sequences are the primary targets of DNA methylation which play an important role in structural and functional alterations in plants. Role of retrotransposons in apomictic development has also been speculated, but exact role of retrotransposon and its differential expression in apomictic and sexual individual are not yet clear. In a study to elucidate the role/involvement of retrotransposons in apomixis in Cenchrus ciliaris, different classes of retrotransposons were identified from the available EST/BAC sequence database of $C$. ciliaris. Expression analysis was performed to see if the retrotransposons are differentially expressed in obligate apomictic and sexual plants. Among the 19 different retrotransposons tested for their expression/activity, six of them were observed to show their activity associated with apomixis in apomictic plants, indicating their possible role in apomixis (Unpublished results).

One of the retrotransposon (C-105) showing $\sim 90 \%$ sequence homology in apomictic and sexual $C$. ciliaris plants was used to determine the role of epigenetic (DNA methylation) regulation, if any, on its differential activity in apomictic and sexual plants. Bisulfite sequencing of the retrotransposon $(\sim 0.3 \mathrm{~Kb}$ fragment $)$ revealed hypermethylation in the region in the sexual plant. More than $95 \%$ cytosine in the fragment analyzed was found to be methylated in sexual plant, compared to only $35 \%$ in apomictic plant. More than 8 -fold increase in methylation in $\mathrm{CHH}$ context, a minor increase in in CG context, but no change in cytosine methylation in CHG context was observed in sexual plant (Figure 1). It is also hypothesized that apomixis represents reversible epigenetic silencing of sexual pathway. Mutant analyses have shown that transition from somatic fate to reproductive fate in Arabidopsis ovules is epigenetically controlled by a sRNA-mediated silencing pathway involving ARGONAUTE 9 (AGO9) protein. ${ }^{64}$ Loss-of-function of agol04 (a maize homolog of AGO9) also resulted in apomixis-like traits, giving rise to $\sim 70 \%$ of functional unreduced female gametes. ${ }^{65}$ Further support for epigenetic regulation of apomixis was provided by comparative analyses of sexual development in maize with the apomixis process in its wild relative apomictic Tripsacum. ${ }^{66}$ The results indicate difference in expression of only a small number of chromatin remodeling enzymes (e.g. CMT3 and DRM2) between apomicts and sexual individuals. Interestingly, features of apospory or diplospory have recently been observed in
Arabidopsis and maize carrying mutant alleles of the genes involved in DNA methylation and siRNA pathways. ${ }^{64,66}$ Podio et al., ${ }^{67}$ reported artificial phenotype reversion in a natural apomictic Paspalum simplex because of epigenetically induced variation in gene expression. Author's studies on epigenetic regulation of apomixis, it was found that certain regions of $C$. ciliaris genome are hypermethylated in obligate sexual plants compared to that in the obligate apomictic plants. ${ }^{68}$ The role of TEs in epigenetic control of the genes associated with apomixis in Cenchrus is also being investigated (Unpublished data). However, causal link between epigenetic mechanism(s) and apomixis is yet to be established. ${ }^{69,70}$ Thus, epigenetic regulation of apomixis is an attractive theory as it potentially accounts for the facultative nature of apomixis as well as the ability of apomicts to revert back to sexuality.

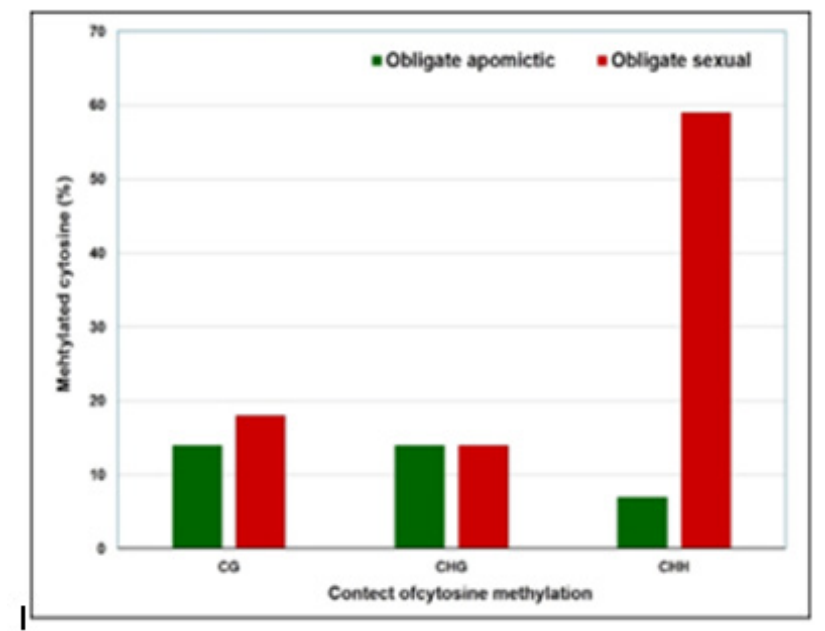

Figure ICytosine methylation in different contexts (CG, $\mathrm{CHG}$ and $\mathrm{CHH}$ contexts) in obligate apomictic and obligate.

\section{Use of apomixis in crop breeding}

Apomixis has tremendous potential applications in agriculture particularly in hybrid seed production for maintaining hybrid vigor over the generations. ${ }^{71}$ Introgression of apomixis from wild relatives to a crop species and transformation of sexual genotype into an apomictic genotype is long-awaited dreams of plant breeders who believe that introduction of apomixis in agronomically important crops will have revolutionary implications. If apomixis can be successfully engineered in a crop plant with a sufficient degree of flexibility, its impact on agriculture could be overwhelming. Apomixis may help fixing the desired genotype immediately in a desired plant, developing hybrids for most of the crops, development, mass production and maintenance of elite parental lines. In addition, it can revolutionize plant breeding procedures by adopting individual plant-based strategies instead of the currently used family-based strategies. It can also provide ecologically sound protection from horizontal transfer of transgenes by introducing autonomous apomixis into male-sterile line. The potential benefits of harnessing apomixis are many including exploitation of heterosis by resowing the best hybrids, clonal propagation of superior genotypes through seeds etc. Apomixis can also be helpful in clonally propagated crops wherein pathogens (mainly endophytic) get accumulate over successive rounds of vegetative propagation and seriously limit the yield/quality of the produce. Apomixis can eliminate expensive/difficult tissue culture procedures used for disease-free multiplication of planting 
material. It can also make storage of planting material easier, and allow saving the produce used as planting material, for example in case of potato, sugarcane etc. Materials used for clonal propagation (e.g. tubers, corms, stem cuttings) have serious disadvantages due to the infection of pathogens, especially viruses. Making the propagation material free from diseases requires cumbersome techniques, while propagation through apomictic seeds can make this easier because seeds carry much fewer plant viruses. Therefore, use of apomictic seeds as multiplication material instead of plant parts is expected to minimize the severity of viral disease. ${ }^{7}$ Fixing a heterozygous genotype using apomixis can make breeding programs faster and cheaper. Apomixis can also provide one of the possible containment strategies for minimizing gene-flow from genetically modified organisms to reduce biosafety issues. ${ }^{72,73}$ Because many apomictic plants produce no viable or compatible pollen, it is possible that apomixis can be used to develop GM plants with reduced risk of gene-flow without compromising seed. Despite numerous advantages mentioned above, apomixis could not be introduced/transferred to a domesticated crop species, as yet. ${ }^{27}$

\section{Conclusion and future perspectives}

Recent data indicate that apomictic seed development in plants is linked to a dynamic pattern of transcriptional activity in ovule probably regulated through epigenetic mechanisms. Epigenetic model of regulation of apomixis is still in infancy and predicts that reversible changes in chromatin configuration might alter the expression of key genes of apomictic pathway at different developmental stage or in different cell types. ${ }^{1}$ The observation that in many apomictic species the developmental program is not tightly conserved, and that differences in the initiation of apomixis in response to environmental conditions/stresses provide evidences to support the view that apomixis is epigenetically regulated. Such flexibility is the characteristic of epigenetic regulatory mechanism. Epigenetic regulation provides a way to achieve desirable variability and adaptive advantages without altering DNA sequence. ${ }^{74}$ Importantly, generation of epialleles can alter the timing of expression of gene(s) controlling cellular or physiological processes during plant development. Inheritance of such adaptive epialleles could provide increased fitness to the plant in the changing environmental conditions and enhanced adaptive flexibility. Identification and functional analysis of several epigenetic regulatory factors involved in gametophyte and seed development process confirm the role of epigenetic regulation in sexual plant reproduction. ${ }^{4,75,76}$ It is expected that future studies on epigenetic regulation of the components of apomixis and sexual seed development would possibly verify whether epigenetic mechanisms really play role in apomixis or not. Unless we understand the regulatory mechanism(s) controlling sexual/ apomictic seed development it would be difficult to transfer/introduce this wonderful trait in crop plant for its successful/sustainable use to enhance crop production. Several groups of scientists are currently working world over to identify the gene(s) involved, to functionally validate the candidate genes in model plant, to establish proof of the concept of introducing apomixis in crop plant as well as to understand the regulatory mechanisms controlling apomixis/sexuality in plant. With the rapidly improving tools and techniques of molecular biology and biotechnology, it is expected that soon we may achieve complete understanding about this amazing biological phenomenon, and we might be able to use it for the benefits of humankind in the near future.

\section{Acknowledgements}

None.

\section{Conflict of interest}

The author declares no conflict of interest.

\section{References}

1. Koltunow AMG, Grossniklaus U. Apomixis: a developmental perspective. Annu Rev Plant Biol. 2003;54:547-574

2. Kumar S, Vishnu Bhat. High-frequency direct plant regeneration via multiple shoot induction in the apomictic forage grass Cenchrus ciliaris L. In Vitro Cell Dev Biol. 2012;48(2):241-248.

3. Kumar S, Saxena S, Gupta MG. Marker-assisted screening of $F_{2}$ mapping population of Cenchrus ciliaris L. segregating for apomictic and sexual mode of reproduction. Crop Breeding and Applied Biotechnology. $2017 ; 17(1): 10-17$.

4. Conner JA, Mookkan M, Huo H, et al. A parthenogenesis gene of apomict origin elicits embryo formation from unfertilized eggs in a sexual plant. Proc Natl Acad Sci U S A. 2015; 112(36):11205-11210.

5. Ozias-Akins P, vanDijk PJ. Mendelian genetics of apomixis in plants Annu Rev Genet. 2007;41:509-537.

6. Kaushal P, Malaviya DR, Roy AK, et al. Reproductive pathways of seed development in apomictic guinea grass (Panicum maximum Jacq.) reveal uncoupling of apomixis components. Euphytica. 2006;164(1):8192.

7. Kandemir N, Saygili I. Apomixis: new horizons in plant breeding. Turk J Agri Forestry. 2015;39.

8. Kumar S, Sheena Saxena. Sequence characterized amplified regions linked with apomictic mode of reproduction in four different apomictic Cenchrus species. Mol Plant Breeding. 2016;7(8):1-14.

9. Carman JG. Asynchronous expression of duplicate genes in angiosper$\mathrm{ms}$ may cause apomixis, bispory, tetraspory and polyembryony. Biological J Linnean Soc. 1997;61(1):51-94.

10. Kumar S, Chandra A, Gupta MG, et al. Molecular and embryological analyses of rare sexual plant in buffelgrass (Cenchrus ciliaris L.). Apomixis research in India: past efforts and future strategies. 2010;31(1):36-40.

11. Voigt-Zielinski M-L, Piwczynski M, Sharbel T. Differential effects of polyploidy and diploidy on fitness of apomictic Boechera. Sex Plant Reprod. 2012;25(2):97-109.

12. Rodriguez-Leal D, Vielle-Calzada J-P. Regulation of apomixis:learning from sexual experience. Curr Opin Plant Biol. 2012;15(5):549-555.

13. Kaushal P, Zadoo SN, Malaviya DR, et al. Apomixis research in India: past efforts and future strategies. Curr Sci. 2012;89(7):1092-1096.

14. Kharrat-Souissi A, Baumel A, Torre F, et al. New insights into the polyploid complex Cenchrus ciliaris L. (Poaceae) show its capacity for gene flow and recombination processes despite its apomictic nature. Aust J Bot. 2011;59:543-553.

15. Hamrick JL, Godt MJW. Allozyme diversity in plant species. In: Plant Population Genetics, Breeding, and Genetic Resources. AHD Brown, et al, editors. Sinauer, Sunderland, MA; 1990. p. 43-63.

16. Adolesson S, Bengtsson BO. The spread of apomixis and its effect on resident genetic variation. J Evol Biol. 2007;20(5):1933-1940.

17. Balloux F, Lehmann L, De Meeus T. The population genetics of clonal and partially clonal diploids. Genetics. 2003;164(4):1635-1644.

18. Bengtsson BO. Genetic variation in organisms with sexual and asexual reproduction. J Evol Biol. 2003;16(2):189-199. 
19. Yadav CB, Anuj, Kumar S, et al. Genetic linkage maps of the chromosomal regions associated with apomictic and sexual modes of reproduction in Cenchrus ciliaris. Mol Breeding. 2012;30(1):239-250.

20. Kumar S. The role of biopesticides in sustainably feeding the nine billion global populations. J Biofertil Biopestici. 2013;4:e114.

21. Albertini E, Barcaccia G, Porceddu A, et al. Mode of reproduction is detected by Parth1 and Sex1 SCAR markers in a wide range of facultative apomictic Kentucky bluegrass varieties. Mol Breeding. 2001;7(4):293300 .

22. Bray RA. Evidence for facultative apomixis in Cenchrus ciliaris. Euphytica. 1978;27(3):801-804.

23. Savidan Y. Chromosomal and embryological analyses in sexual $\mathrm{x}$ apomictic hybrids of Panicum maximum Jacq. Theor Appl Genet. 1980;58(2):153-156.

24. Kumar S, Chandra A, Gupta MG, et al. IGFRI-CcSx-08/1 (IC0590889; INGR11062), an Anjan grass (Cenchrus ciliaris L.) germplasm with a rare obligate sexual plant. Ind J Plant Genet Resou. 2013;26:99-100.

25. Quarin CL, Burson BL. Cytology of sexual and apomictic Paspalum species. Cytologia. 1991;56(2):223-228.

26. Hanna W, Dujardin M, Ozias-Akins P, et al. Reproduction, cytology, and fertility of Pearl millet $\times$ Pennisetum squamulatum $\mathrm{BC} 4$ plants. $J \mathrm{He}$ red. 1993;84(3):213-216.

27. Barcaccia G, Albertini E. Apomixis in plant reproduction:a novel perspective on an old dilemma. Plant Reprod. 2013;26(3):159-179.

28. Ozias-Akins P, Roche D, Hanna WW. Tight clustering and hemizygosity of apomixis-linked molecular markers in Pennisetum squamulatum implies genetic control of apospory by a divergent locus that may have no allelic form in sexual genotypes. Proc Natl Acad Sci US A. 1998;95(9):5127-5132.

29. Pessino SC, Ortiz J, Leblanc O, et al. Identification of a maize linkage group related to apomixis in Brachiaria. Theoretical and Applied Genetics. 1997;94(3-4):439-444.

30. Martinez EJ, Hopp HE, Stein J, et al. Genetic characterization of apospory in tetraploid Paspalum notatum based on the identification of linked molecular markers. Molecular Breeding. 2003;12(4):319-327.

31. Grimanelli D, Leblanc O, Espinosa E, et al. Mapping diplosporous apomixis in tetraploid Tripsacum: one gene or several genes?. Heredity (Edinb). 1998;80(1):33-39.

32. Kumar S. Developments in molecular markers for genetic analysis of plants. In: Recent Advances in Plant Technology. A Kumar, ed. Jaipur, India: Pointer Publishers; 2010. p. 186-211.

33. Chandra A, Tiwari KK, Nagaich D, et al. Development and characterization of microsatellite markers from tropical forage Stylosanthes species, analysis of genetic variability and cross-species transferability. Genome. 2011;54(12):1016-1028.

34. Goel S, Chen Z, Conner JA, et al. Physical evidence that a single hemizygous chromosomal region is sufficient to confer aposporous embryosac formation in Pennisetum squamulatum and Cenchrus ciliaris. Genetics. 2003;163:1069-1082.

35. Dwivedi KK, Bhat SR, Bhat V, et al. Identification of a SCAR marker linked to apomixis in buffelgrass (Cenchrus ciliaris L.). Plant Science. 2007;172(4):788-795.

36. Barcaccia G, Mazzucato A, Albertini E, et al. Inheritance of parthenogenesis in Poa pratensis L:auxin test and AFLP linkage analyses support monogenic control. Theoretical and Applied Genetics. 1998;97(12):74-82.
37. Savidan Y. Genetics and utilization of apomixis for the improvement of guinea grass (Panicum maximum Jacq.). In: Proceedings of International Grassland Congress. (JA Smith, VW Hays, Eds) Lexington, KY; 1983. p. 182-184.

38. Gustine DL, Sherwood RT, Huff DR. Apospory-linked molecular markers in buffel grass. Crop Sci. 1997;37:947-951.

39. Roche D, Peisheng C, Zhenbang C, et al. An apospory-specific genomic region is conserved between buffelgrass (Cenchrus ciliaris L.) and Pennisetum squamulatum Fresen. Plant J. 1999;19:203-208.

40. Leblanc O, Grimanelli D, de Leon, et al. Detection of the apomictic mode of reproduction in maize-Tripsacum hybrids using maize RFLP markers. Theor Appl Genet. 1995;90(7-8):1198-1203.

41. Tucker, M, Ana-Claudia GA, Nocholas AP, et al. Sexual and apomictic reproduction in Hieracium subgenus Pilosella are closely interrelated developmental pathways. Plant Cell. 2003;15:1524-1537.

42. Dwivedi KK, Radhakishna A, Kumar S, et al. Development of an ISSRderived SCAR marker linked to apospory in buffel grass (Cenchrus ciliaris L.). Ind J Gen Plant Breeding. 2015;75(2):271-273.

43. Kumar S, Chandra A, Gupta MG, et al. SCAR marker linked to sexuality in apomictic buffelgrass (Cenchrus ciliaris L.). Range Mgmt Agroforestry. 2010;31:149-150.

44. Kumar S. RNAi (RNA interference) vectors for functional genomics study in plants. National Academy Science Letters. 2014;37(3):289-294.

45. Batra S, Kumar S. In vitro high frequency plant regeneration in buffelgrass (Cenchrus ciliaris L.). J Plant Biol. 2002;29:191-194.

46. Kumar S, Bhat V. High-frequency direct plant regeneration via multiple shoot induction in the apomictic forage grass Cenchrus ciliaris L. In Vitro Cellular \& Developmental Biology - Plant. 2012;48:241-248.

47. Kumar S, Sahu N, Singh A. High-frequency in vitro plant regeneration via callus induction in a rare sexual plant of Cenchrus ciliaris L. In Vitro Cellular \& Developmental Biology - Plant. 2015;51(1):28-34.

48. Bhat V, Dalton SJ, Kumar S, et al. Particle-inflow-gun-mediated genetic transformation of buffel grass (Cenchrus ciliaris L.):optimizing biological and physical parameters. J Appl Genet. 2001;42(4):405 - 412.

49. Batra S, Kumar S. Agrobacterium-mediated transient GUS gene expression in buffel grass (Cenchrus ciliaris L.). J Appl Genet. 2003;44(4):449-458.

50. Sharbel TF, Voigt ML, Corral JM, et al. Apomictic and sexual ovules of Boechera display heterochronic global gene expression patterns. Plant Cell. 2010;22:655-671.

51. Schmidt A, Schmid MW, Klostermeier UC, et al. Apomictic and sexual germline development differ with respect to cell cycle, transcriptional, hormonal and epigenetic regulation. PLoS Genet. 2014;10:e1004476.

52. Kumar S, Singh A. Epigenetic regulation of abiotic stress tolerance in plants. Adv Plants Agric Res. 2016;5:e00179.

53. Kumar S, Beena AS, Awana M, et al. Salt-induced tissue-specific cytosine methylation downregulates expression of $H K T$ genes in contrasting wheat (Triticum aestivum L.) genotypes. DNA Cell Biol. 2017;36:e3505.

54. Yan HH, Kikuchi S, Neumann P, et al. Genome wide mapping of cytosine methylation revealed dynamic DNA methylation patterns associated with genes and centromeres in rice. Plant J. 2010;63:353-365.

55. Feng S, Cokus SJ, Zhang X, et al. Conservation and divergence of methylation patterning in plants and animals. Proc Natl Acad Sci USA. 2010;107:8689-8694.

56. Wang X, Li Q, Yuan W, et al. The cytosolic Fe-S cluster assembly component MET18 is required for the full enzymatic activity of ROS1 in active DNA demethylation. Sci Rep. 2016;6:e26443. 
57. Zhang K, Sridhar VV, Zhu J, et al. Distinctive core histone post-translational modification patterns in Arabidopsis thaliana. PLOS ONE. 2007;2:e1210.

58. Camporeale G, Oommen AM, Griffin JB, et al. $\mathrm{K}_{12}$-biotinylated histone $\mathrm{H}_{4}$ marks heterochromatin in human lymphoblastoma cells. J Nutr Biochem. 2007;18:760-768.

59. Chinnusamy V, Zhu JK. Epigenetic regulation of stress responses in plants. Curr Opin Plant Biol. 2009;12:133-139.

60. Borsani O, Zhu J, Verslues PE, et al. Endogenous siRNAs derived from a pair of natural $\mathrm{cis}$-antisense transcripts regulate salt tolerance in $\mathrm{Ara}$ bidopsis. Cell. 2005;123:1279-1291.

61. Ha HC, Sirisoma NS, Kuppusamy P, et al. The natural polyamine spermidine functions directly as free radical scavenger. Proc Natl Acad Sci USA. 1998;95:11140-11145.

62. Conner JA, Goel S, Gunawan G, et al. Sequence analysis of bacterial artificial chromosome clones from the apospory-specific genomic region of Pennisetum and Cenchrus. Plant Physiol. 2008;147:1396-1411.

63. Horandl E, Hadacek F. The oxidative damage initiation hypothesis for meiosis. Plant Reprod. 2013;26:351-367.

64. Olmedo-Monfil V, Dura'n-Figueroa N, Arteaga-Va'zquez M, et al Control of female gamete formation by a small RNA pathway in Arabidopsis. Nature. 2010;464:628-632.

65. Singh M, Goel S, Meeley RB, et al. Production of viable gametes without meiosis in maize deficient for an ARGONAUTE protein. Plant Cell. 2011;23:443-458.

66. Garcia-Aguilar M, Michaud C, Leblanc O, et al. Inactivation of a DNA methylation pathway in maize reproductive organs results in apomixislike phenotypes. Plant Cell. 2010;22:3249-3267.
67. Podio M, Cáceres ME, Samoluk SS, et al. A methylation status analysis of the apomixis-specific region in Paspalum spp. suggests an epigenetic control of parthenogenesis. J Exp Bot. 2014;65:6411-6424.

68. Kumar S, Bhat V. Application of omics technologies in forage crop improvement. In: Omics Applications in Crop Science (D Barh. Ed). CRC Press, USA: Taylor \& Francis LLC; 2014. p. 523-548.

69. Amiteye S, Corral JM, Vogel H, et al. Analysis of conserved microRNAs in floral tissues of sexual and apomictic Boechera species. BMC Genomics. 2011;12:e500.

70. Galla G, Volpato M, Sharbel TF, et al. Computational identification of conserved microRNAs and their putative targets in Hypericum perforatum L. flower transcriptomes. Plant Reprod. 2013;26:209-229. doi:10.1007/s00497-013-0227-6.

71. Koltunow AM, Tucker MR. Functional embryosac formation in Arabidopsis without meiosis-one step towards asexual seed formation (apomixis) in crops? J Biosci. 2008;33:309-311.

72. Daniell H. Molecular strategies for gene containment in transgenic crops. Nat Biotechnol. 2002;20:581-586.

73. Kumar S. Biosafety issues of genetically modified organisms. Biosafety. 2014;3:e150. doi:10.4172/2167-0331.1000e150.

74. Rodrigues JCM, Koltnow MAG. Epigenetic aspects of sexual and asexual seed development. Acta Biol Craco Series Botanica. 2005;47:37-49.

75. Shi J, Ding A, Shen W-H. Epigenetic regulation of rice flowering and reproduction. Front Plant Sci. 2014;5:e803.

76. Kawashima T, Berger F. Epigenetic reprogramming in plant sexual reproduction. Nat Rev Genet. 2014;15:613-624. 